\title{
Hydrodynamics of topological Dirac semi-metals with chiral and $\mathbb{Z}_{2}$ anomalies
}

\author{
Marek Rogatko and Karol I. Wysokinski \\ Institute of Physics, Maria Curie-Sktodowska University, \\ pl. Marii Curie-Sktodowskiej 1, Lublin, 20-031 Poland \\ E-mail: rogat@kft.umcs.lublin.pl, karol@tytan.umcs.lublin.pl
}

ABSTRACT: We consider the hydrodynamical model of topological Dirac semi-metal possessing two Dirac nodes separated in momentum space along a rotation axis. It has been argued that the system in question, except the chiral anomaly, is endowed with the other one $\mathbb{Z}_{2}$. In order to model such a system we introduce two U(1)-gauge fields. The presence of the additional $\mathbb{Z}_{2}$ anomaly leads to the non-trivial modifications of hydrodynamical equations and to the appearance of new kinetic coefficients bounded with the vorticity and the magnetic parts of Maxwell and auxiliary U(1)-gauge fields.

KEYwORDS: Holography and condensed matter physics (AdS/CMT), Anomalies in Field and String Theories, Holography and quark-gluon plasmas

ARXIV EPRINT: 1804.02202 


\section{Contents}

1 Introduction 1

2 Hydrodynamical model 3

3 Application to Dirac semi-metals with $\mathbb{Z}_{2}$ topological charge 9

4 Summary and conclusions $\quad 13$

\section{Introduction}

The possibility of the hydrodynamic approach to transport relies on the fact that strong interactions of the constituent particles which, at low energies and long length-scales, move like a fluid can be described with only a few collective or slowly varying variables. These include the local velocity $v(x)$, temperature $T(x)$ and chemical potentials $\mu_{a}(x)$ related to all conserved charges (their densities are denoted by $\rho_{a}(x)$ ). The hydrodynamics of the relativistic fluid has been developed by Landau [1] and others [2] and generalized to take relativistic triangle anomalies $[3,4]$ into account.

A purely hydrodynamic derivation of the anomaly effects, considering the first order in derivation expansion was presented in [5]. The idea was to examine the local entropy production rate in the presence of anomalies and impose the positivity constraint stemming from the second law of thermodynamics. It was shown that the contributions from the anomaly to the entropy production were locally unbounded and might potentially violate the second law of thermodynamics, so the proper generalizations were necessary. In turn, these facts lead to a set of differential equations for the novel transport coefficients connected with the anomaly. Further, this idea was implemented to the case of anomalous superfluids [6]-[8] and non-abelian symmetry $[9,10]$.

On the other hand, the chiral magnetic anomaly, i.e., anomaly induced phenomenon of electric charge separation along the axis of the applied magnetic field in the presence of fluctuating topological charge was widely studied [11]-[15]. The chiral magnetic effect in hydrodynamical approach, in a model with two conserved currents, vector and axial and the associated chemical potentials was studied in [16]. The aforementioned phenomenon has attracted a lot of attention due to the possible explanation of an experimentally observed charge asymmetry in heavy ion collisions and provided explanation for the observed decay of neutral pion into photons.

The anomalies have been predicted [17] and later found [18, 19] to play an important part in the description of electrons in solids. Recently, the hydrodynamics with vector and axial currents and the holographic charged fluid with electric separation effect was paid 
attention to [20]. It turns out that the gravitational backreaction effect may be responsible for the emergence of an axial current as a response to an axial electric one.

The necessity of relativistic description of electrons in solids may appear superficial, as the velocity of electrons in solids typically equals a small fraction of the light velocity. However, the spectrum of electrons in many materials and close to some special points in the Brillouin zone, has a relativistic form characteristic for massless particles. Such Dirac — like massless nature of spectrum is protected by symmetries and has been spotted in the twodimensional graphene [21] and at the surfaces of the crystalline topological insulators [22]. The Dirac - like spectrum is predicted and observed in the three-dimensional materials known as Dirac or Weyl semi-metals [23-29]. The transport properties of graphene with the Dirac point at the Fermi energy have been proposed to follow the hydrodynamic description [30]. Later measurements confirmed the hydrodynamic behavior of electrons in graphene [31] and in three-dimensional systems [28, 29, 32-34]. All this makes the relativistic hydrodynamic approach to electrons in condensed matter a timely and important issue.

Moreover, the recent experimental works provide clear evidences that chiral anomaly is observed in condensed matter systems. Namely, it was spotted in Dirac semi-metal $\mathrm{Na}_{3} \mathrm{Bi}$ [35], $\mathrm{ZrTe}_{5}$ [36], as well as, in Weyl semi-metal TaAs and NbP [37]-[39]. The mentioned two classes of Dirac semi-metals (DSM) have acquired attention in the contemporary investigations. In the first one the Dirac points appear at the time reversal invariant momenta in the first Brillouin zone, while in the other the Dirac points take place in pairs and are separated in momentum space along a rotational axis [40, 41]. It turns out that the experimentally found examples of DSM belong mainly to the second class of the aforementioned materials.

The Dirac points in the second class of aforementioned semi-metals are endowed with a non-trivial $\mathbb{Z}_{2}$ topological invariant protecting the nodes and leading to the presence of Fermi arc surface states [42]-[45]. The novel charge, in a close analogy to the chiral one, is also not conserved under the action of external fields. The non-conservation of the novel anomalous charge has been argued to have an effect on transport characteristics of materials [46]. Thus the recent studies of three dimensional condensed matter systems open the doors to symmetries not spotted in other relativistic objects, making the subject even more intriguing.

The main motivation behind our considerations is a natural question about the possible influence of $\mathbb{Z}_{2}$ topological charge on transport characteristics of the studied materials. The aim of the present work is to generalize relativistic hydrodynamics including the chiral anomaly $[3,4]$ and the additional anomaly, which we call $\mathbb{Z}_{2}$ anomaly after the paper [46]. The two anomalous charges in the considered theory require the existence of the two conjugate to them chemical potentials $\left(\mu\right.$ and $\left.\mu_{d}\right)$. At the equilibrium both chemical potentials take zero values. Accordingly we also introduce two U(1)-gauge fields, one being the standard Maxwell field coupled to the chiral anomalous charge and other coupled to the $\mathbb{Z}_{2}$ topological charge. The derived set of hydrodynamic equations generalizes those previously found [5] and extensively discussed [47, 48] in the literature.

The organization of the paper is as follows. In the next section 2 we present the calculations leading to generalization of the relativistic hydrodynamic equations [5] in such 
a way that they take into account two anomalous charges, responsible for chiral and $\mathbb{Z}_{2}$ anomalies. In section 3 we conclude with the discussion of the main results and possible modifications of the transport characteristics of materials.

\section{Hydrodynamical model}

In this section we examine the hydrodynamical model of topological Dirac semi-metal in which two Dirac nodes, protected by rotational symmetry, are separated in momentum space along a rotation axis. It has been argued that the aforementioned system constitutes a source of the additional $\mathbb{Z}_{2}$ anomaly, except the chiral one, which leads to the nonconservation of the corresponding anomalous $\mathbb{Z}_{2}$ topological charge [46]. In order to model such a system we consider anomalous charges connected with two U(1)-gauge fields. One of them is the ordinary Maxwell gauge field, the other is the additional one connected with the $\mathbb{Z}_{2}$ anomalous charge. The hydrodynamical equations of motion in the presence of $\mathbb{Z}_{2}$ and chiral anomalies are provided by

$$
\begin{aligned}
\partial_{\alpha} T^{\alpha \beta}(F, B) & =F^{\beta \alpha} j_{\alpha}(F)+B^{\beta \alpha} j_{\alpha}(B), \\
\partial_{\alpha} j^{\alpha}(F) & =C_{1} E_{(F) \alpha} B^{(F) \alpha}+C_{2} \tilde{E}_{(B) \alpha} \tilde{B}^{(B) \alpha}, \\
\partial_{\alpha} j^{\alpha}(B) & =C_{3} \tilde{E}_{(B) \alpha} B^{(F) \alpha}+C_{4} E_{(F) \alpha} \tilde{B}^{(B) \alpha},
\end{aligned}
$$

where $C_{i}, i=1, \ldots, 4$ denote the constants which determine the adequate anomalies. The electric and magnetic components of the two gauge fields, in the fluid rest frame, are written respectively as

$$
\begin{array}{ll}
E_{\alpha}^{(F)}=F_{\alpha \beta} u^{\beta}, & B_{\alpha}^{(F)}=\frac{1}{2} \epsilon_{\alpha \beta \rho \delta} u^{\beta} F^{\rho \delta}, \\
\tilde{E}_{\alpha}^{(B)}=B_{\alpha \beta} u^{\beta} & \tilde{B}_{\alpha}^{(B)}=\frac{1}{2} \epsilon_{\alpha \beta \rho \delta} u^{\beta} B^{\rho \delta} .
\end{array}
$$

$F_{\alpha \beta}=2 \partial_{[\alpha} A_{\beta]}$ stands for the ordinary Maxwell field strength tensor, while the second U(1)gauge field $B_{\alpha \beta}$ is given by $B_{\alpha \beta}=2 \partial_{[\alpha} B_{\beta]}$. On the other hand, $j_{\alpha}(F), j_{\alpha}(B)$ represent the adequate currents connected with the gauge fields. The relation (2.2) describes the modifications of the anomalous chiral charge conservation law when the external magnetic and electric fields parallel to each other are applied to the system, while the equation (2.3) expresses the changes of the anomalous $\mathbb{Z}_{2}$ charge conservation law.

The energy momentum tensor and the currents needed for the hydrodynamic description of the relativistic fluid are given by $[1,5]$

$$
\begin{aligned}
T^{\alpha \beta} & =(\epsilon+p) u^{\alpha} u^{\beta}+p g^{\alpha \beta}+\tau^{\alpha \beta}, \\
j^{\alpha}(F) & =\rho u^{\alpha}+V_{F}^{\alpha}, \\
j^{\alpha}(B) & =\rho_{d} u^{\alpha}+V_{B}^{\alpha},
\end{aligned}
$$

where $\epsilon$ is the energy per unit volume, $p$ the pressure of the fluid, $\rho, \rho_{d}$ are the $\mathrm{U}(1)$ charge densities, while $\tau^{\alpha \beta}$ and $V_{F(B)}^{\alpha}$ depict higher order corrections in velocity gradients and correspond to the dissipative effects in the fluid. In the rest frame of the fluid element, 
there are no dissipative forces and $u_{\alpha} \tau^{\alpha \beta}=0$ and $u_{\alpha} V_{F}^{\alpha}=u_{\alpha} V_{B}^{\alpha}=0$. The four-vector $u^{\alpha}$, with the normalization $u_{\alpha} u^{\alpha}=-1$, describes the flow of the considered fluid.

Using the thermodynamical relations

$$
\epsilon+p=T s+\mu \rho+\mu_{d} \rho_{d}, \quad d p=s d T+\rho d \mu+\rho_{d} d \mu_{d},
$$

where $s$ is the entropy per unit volume, the explicit expression for energy-momentum tensor and $u_{\beta} \partial_{\alpha} T^{\alpha \beta}$, as well as, the expressions for $\partial_{\alpha} j^{\alpha}(F)$ and $\partial_{\alpha} j^{\alpha}(B)$, one arrives at the following relation:

$$
\begin{aligned}
\partial_{\alpha} s^{\alpha}= & \partial_{\alpha}\left[s u^{\alpha}-\frac{\mu}{T} V_{F}^{\alpha}-\frac{\mu_{d}}{T} V_{B}^{\alpha}\right]=-\frac{1}{T} \tau^{\alpha \beta} \partial_{\alpha} u_{\beta} \\
& -V_{F}^{\alpha}\left[\partial_{\alpha}\left(\frac{\mu}{T}\right)-\frac{E_{\alpha}^{(F)}}{T}\right]-V_{B}^{\alpha}\left[\partial_{\alpha}\left(\frac{\mu_{d}}{T}\right)-\frac{\tilde{E}_{\alpha}^{(B)}}{T}\right] \\
& -\frac{\mu}{T}\left(C_{1} E_{\alpha}^{(F)} B^{(F) \alpha}+C_{2} \tilde{E}_{\alpha}^{(B)} \tilde{B}^{(B) \alpha}\right)-\frac{\mu_{d}}{T}\left(C_{3} \tilde{E}_{\alpha}^{(B)} B^{(F) \alpha}+C_{4} E_{\alpha}^{(F)} \tilde{B}^{(B) \alpha}\right),
\end{aligned}
$$

where in our system we define

$$
\begin{aligned}
& V_{F}^{\alpha}=-\sigma_{F}\left[T P^{\alpha \beta} \partial_{\beta}\left(\frac{\mu}{T}\right)-E^{(F) \alpha}\right]-\sigma_{F \tilde{B}}\left[T P^{\alpha \beta} \partial_{\beta}\left(\frac{\mu_{d}}{T}\right)-\tilde{E}^{(B) \alpha}\right], \\
& V_{B}^{\alpha}=-\sigma_{B}\left[T P^{\alpha \beta} \partial_{\beta}\left(\frac{\mu_{d}}{T}\right)-\tilde{E}^{(B) \alpha}\right]-\sigma_{B F}\left[T P^{\alpha \beta} \partial_{\beta}\left(\frac{\mu}{T}\right)-E^{(F) \alpha}\right],
\end{aligned}
$$

where the symbol $P^{\alpha \beta}=g^{\alpha \beta}+u^{\alpha} u^{\beta}$ stands for the projector orthogonal to the four-velocity $u^{\alpha}$. As was pointed out in [5], if we did not take into account the influence of the anomalies (i.e., $C_{i}=0$ ), one can interpret the equation (2.10) as the relation describing the entropy production. We ought to have that the right-hand side of it should be greater or equal to zero. This condition leads to the reqiurement imposed on $\sigma_{F}, \sigma_{F \tilde{B}}, \sigma_{B F}$ and $\sigma_{B}$. It can be shown by the direct calculations, that the proviso $\partial_{\alpha} s^{\alpha} \geq 0$, will be satisfied if

$$
\sigma_{F} \geq 0, \quad \sigma_{F} \sigma_{B}-\sigma_{F \tilde{B}} \sigma_{B F} \geq 0, \quad \sigma_{B} \geq 0 .
$$

The above demands originate from the Sylvester criterion for the positivity of the quadratic form, to which one can rewrite the right-hand side of the relation (2.10). Moreover, we supposed positivity of viscosity parameters $\eta$ and $\zeta$ [1] entering the formula for $\tau^{\alpha \beta}$.

Thus, the equation (2.10) can be interpreted as describing the entropy production. Its right-hand side is greater or equal to zero, as required by the second law of thermodynamics. The presence of anomalies changes the situation drastically. The terms with $C_{i} \neq 0$ can have either sign and, when negative, can even overcome the rest of the terms appearing in the equation (2.10) and thus spoil the positivity of entropy production. Therefore, the entropy flux $s^{\alpha}$, as well as, all the dissipative terms contributing to the transport current have to be modified.

The most general modification of the entropy current, which comprises standard dissipation terms, vorticity $\omega_{\alpha}=(1 / 2) \epsilon_{\alpha \beta \rho \delta} u^{\beta} \partial^{\rho} u^{\delta}$ and the terms proportional to the magnetic components of the two $\mathrm{U}(1)$-gauge fields are taken in the form

$$
s^{\alpha}=s u^{\alpha}-\frac{\mu}{T} V_{F}^{\alpha}-\frac{\mu_{d}}{T} V_{B}^{\alpha}+D \omega^{\alpha}+D_{B} B^{(F) \alpha}+D_{\tilde{B}} \tilde{B}^{(B) \alpha} .
$$


The dissipative contribution to the $\mathrm{U}(1)$-gauge field currents are also modified by new transport coefficients $\xi, \xi_{B}, \xi_{d}, \xi_{\tilde{B}}, \xi_{F \tilde{B}}$ and $\xi_{\tilde{B} F}$

$$
\begin{aligned}
V_{F}^{\alpha}= & -\sigma_{F}\left[T P^{\alpha \beta} \partial_{\beta}\left(\frac{\mu}{T}\right)-E^{(F) \alpha}\right]-\sigma_{F \tilde{B}}\left[T P^{\alpha \beta} \partial_{\beta}\left(\frac{\mu_{d}}{T}\right)-\tilde{E}^{(B) \alpha}\right]+\xi \omega^{\alpha} \\
& +\xi_{B} B^{(F) \alpha}+\xi_{F \tilde{B}} \tilde{B}^{(B) \alpha} \\
V_{B}^{\alpha}= & -\sigma_{B}\left[T P^{\alpha \beta} \partial_{\beta}\left(\frac{\mu_{d}}{T}\right)-\tilde{E}^{(B) \alpha}\right]-\sigma_{B F}\left[T P^{\alpha \beta} \partial_{\beta}\left(\frac{\mu}{T}\right)-E^{(F) \alpha}\right]+\xi_{d} \omega^{\alpha} \\
& +\xi_{\tilde{B}} \tilde{B}^{(B) \alpha}+\xi_{\tilde{B} F} B^{(F) \alpha} .
\end{aligned}
$$

The unknown functions $\xi, \xi_{d}, \xi_{B}, \xi_{\tilde{B}}, \xi_{F \tilde{B}}, \xi_{\tilde{B} F} D, D_{B}, D_{\tilde{B}}$ depend on $T$ and $\mu, \mu_{d}$. Our aim is to find the general formula for these new transport coefficients induced by the quantum anomalies.

Assuming that all the anomaly coefficients $C_{i} \neq 0$, one finds the conditions required for the positivity of $\partial_{\alpha} s^{\alpha}$. During the direct calculations of the divergence of the entropy current, one encounters the derivatives of the vorticity $\partial_{\alpha} \omega^{\alpha}$ to the vorticity $\omega^{\alpha}$ itself and similarly, the $\partial_{\alpha} B^{\alpha}$ is related to $B^{\alpha}$. For our hydrodynamics (linear in the derivatives of velocity) it is enough to find the required relations for the ideal fluid. They may be achieved by projecting the underlying equations of motion (2.6)-(2.8) of the hydrodynamical model along two orthogonal directions. Namely, along $u^{\alpha}$ and $P_{\beta}^{\alpha}=\delta_{\beta}^{\alpha}+u^{\alpha} u_{\beta}$. As a result we achieve the following relations for the ideal hydrodynamics (i.e., with $\tau^{\alpha \beta}=0$, $\left.V_{F}^{\alpha}=V_{B}^{\alpha}=0\right)$

$$
\begin{aligned}
\partial_{\alpha} \omega^{\alpha} & =\frac{2 \omega_{\alpha}}{\epsilon+p}\left(-\partial^{\alpha} p+F^{\alpha \beta} j_{\beta}(F)+B^{\alpha \beta} j_{\beta}(B)\right), \\
\partial_{\alpha} B^{(F) \alpha} & =-2 \omega_{\alpha} E^{(F) \alpha}+\frac{B_{\alpha}^{(F)}}{\epsilon+p}\left(-\partial^{\alpha} p+F^{\alpha \beta} j_{\beta}(F)+B^{\alpha \beta} j_{\beta}(B)\right), \\
\partial_{\alpha} \tilde{B}^{(B) \alpha} & =-2 \omega_{\alpha} \tilde{E}^{(B) \alpha}+\frac{\tilde{B}_{\alpha}^{(B)}}{\epsilon+p}\left(-\partial^{\alpha} p+F^{\alpha \beta} j_{\beta}(F)+B^{\alpha \beta} j_{\beta}(B)\right) .
\end{aligned}
$$

We evaluate of $\partial_{\alpha} s^{\alpha}$ with the help of (2.17)-(2.19). However, the resulting expression comprises a number of terms containing $\omega^{\alpha}, B^{(F) \alpha}, \tilde{B}^{(B) \alpha}, \omega^{\alpha} E_{\alpha}^{(F)}, \omega^{\alpha} \tilde{E}_{\alpha}^{(B)}, E_{\alpha}^{(F)} B^{(F) \alpha}$, $\tilde{E}_{\alpha}^{(B)} \tilde{B}^{(B) \alpha}, \tilde{E}_{\alpha}^{(B)} B^{(F) \alpha}, E_{\alpha}^{(F)} \tilde{B}^{(B) \alpha}$. The condition $\partial_{\alpha} s^{\alpha} \geq 0$ demands vanishing all factors multiplying the above terms. It eventuates in the following differential equations

$$
\begin{aligned}
\partial_{\alpha} D-2 \frac{\partial_{\alpha} p}{\epsilon+p} D-\xi \partial_{\alpha}\left(\frac{\mu}{T}\right)-\xi_{d} \partial_{\alpha}\left(\frac{\mu_{d}}{T}\right) & =0, \\
\partial_{\alpha} D_{B}-\frac{\partial_{\alpha} p}{\epsilon+p} D_{B}-\xi_{B} \partial_{\alpha}\left(\frac{\mu}{T}\right)-\xi_{\tilde{B} F} \partial_{\alpha}\left(\frac{\mu_{d}}{T}\right) & =0, \\
\partial_{\alpha} D_{\tilde{B}}-\frac{\partial_{\alpha} p}{\epsilon+p} D_{\tilde{B}}-\xi_{\tilde{B}} \partial_{\alpha}\left(\frac{\mu_{d}}{T}\right)-\xi_{F \tilde{B}} \partial_{\alpha}\left(\frac{\mu}{T}\right) & =0,
\end{aligned}
$$

and the additional conditions

$$
\begin{aligned}
\frac{2 D \rho}{\epsilon+p}-2 D_{B}+\frac{1}{T} \xi & =0 \\
\frac{2 D \rho_{d}}{\epsilon+p}-2 D_{\tilde{B}}+\frac{1}{T} \xi_{d} & =0
\end{aligned}
$$




$$
\begin{array}{r}
\frac{\rho D_{B}}{\epsilon+p}+\frac{\xi_{B}}{T}-\mu \frac{C_{1}}{T}=0, \\
\frac{\rho_{d} D_{\tilde{B}}}{\epsilon+p}+\frac{\xi_{\tilde{B}}}{T}-\mu \frac{C_{2}}{T}=0, \\
\frac{\rho D_{\tilde{B}}}{\epsilon+p}-\mu_{d} \frac{C_{4}}{T}+\frac{\xi_{F \tilde{B}}}{T}=0, \\
\frac{\rho_{d} D_{B}}{\epsilon+p}-\mu_{d} \frac{C_{3}}{T}+\frac{\xi_{\tilde{B} F}}{T}=0 .
\end{array}
$$

The differential equations (2.20)-(2.22) suggest the dependence of the parameters $D_{i}=D$, $D_{B}, D_{\tilde{B}}$ on the pressure $p$ and the normalized chemical potentials $\tilde{\mu}=\mu / T$ and $\tilde{\mu}_{d}=\mu_{d} / T$. To exploit this fact we use thermodynamic relations

$$
\begin{aligned}
\partial_{\alpha} D & =\left(\frac{\partial D}{\partial p}\right)_{\tilde{\mu}, \tilde{\mu}_{d}} \partial_{\alpha} p+\left(\frac{\partial D}{\partial \tilde{\mu}}\right)_{p, \tilde{\mu}_{d}} \partial_{\alpha} \tilde{\mu}+\left(\frac{\partial D}{\partial \tilde{\mu}_{d}}\right)_{p, \tilde{\mu}} \partial_{\alpha} \tilde{\mu}_{d}, \\
\partial_{\alpha} D_{B} & =\left(\frac{\partial D_{B}}{\partial p}\right)_{\tilde{\mu}_{,}, \tilde{\mu}_{d}} \partial_{\alpha} p+\left(\frac{\partial D_{B}}{\partial \tilde{\mu}}\right)_{p, \tilde{\mu}_{d}} \partial_{\alpha} \tilde{\mu}+\left(\frac{\partial D_{B}}{\partial \tilde{\mu}_{d}}\right)_{p, \tilde{\mu}} \partial_{\alpha} \tilde{\mu}_{d}, \\
\partial_{\alpha} D_{\tilde{B}} & =\left(\frac{\partial D_{\tilde{B}}}{\partial p}\right)_{\tilde{\mu}, \tilde{\mu}_{d}} \partial_{\alpha} p+\left(\frac{\partial D_{\tilde{B}}}{\partial \tilde{\mu}}\right)_{p, \tilde{\mu}_{d}} \partial_{\alpha} \tilde{\mu}+\left(\frac{\partial D_{\tilde{B}}}{\partial \tilde{\mu}_{d}}\right)_{p, \tilde{\mu}} \partial_{\alpha} \tilde{\mu}_{d},
\end{aligned}
$$

and require vanishing of the coefficients multiplying $\partial_{\alpha} p, \partial_{\alpha} \tilde{\mu}$ and $\partial_{\alpha} \tilde{\mu}_{d}$, which can be considered as having arbitrary values at the initial time slice [5]. This leads to three sets of the differential equations. The first defines the parameter $D\left(p, \tilde{\mu}, \tilde{\mu}_{d}\right)$

$$
\begin{array}{r}
\left(\frac{\partial D}{\partial p}\right)_{\tilde{\mu}, \tilde{\mu}_{d}}-\frac{2 D}{\epsilon+p}=0 \\
\left(\frac{\partial D}{\partial \tilde{\mu}}\right)_{p, \tilde{\mu}_{d}}-\xi=0 \\
\left(\frac{\partial D}{\partial \tilde{\mu}_{d}}\right)_{p, \tilde{\mu}}-\xi_{d}=0
\end{array}
$$

while the next two give the dependence of the partial derivatives of $D_{B}\left(p, \tilde{\mu}, \tilde{\mu}_{d}\right)$

$$
\begin{aligned}
\left(\frac{\partial D_{B}}{\partial p}\right)_{\tilde{\mu}, \tilde{\mu}_{d}}-\frac{D_{B}}{\epsilon+p} & =0 \\
\left(\frac{\partial D_{B}}{\partial \tilde{\mu}}\right)_{p, \tilde{\mu}_{d}}-\xi_{B} & =0 \\
\left(\frac{\partial D_{B}}{\partial \tilde{\mu}_{d}}\right)_{p, \tilde{\mu}}-\xi_{\tilde{B} F} & =0
\end{aligned}
$$


and $D_{\tilde{B}}\left(p, \tilde{\mu}, \tilde{\mu}_{d}\right)$

$$
\begin{aligned}
\left(\frac{\partial D_{\tilde{B}}}{\partial p}\right)_{\tilde{\mu}_{d}, \tilde{\mu}}-\frac{D_{\tilde{B}}}{\epsilon+p} & =0 \\
\left(\frac{\partial D_{\tilde{B}}}{\partial \tilde{\mu}}\right)_{p, \tilde{\mu}_{d}}-\xi_{F \tilde{B}} & =0 \\
\left(\frac{\partial D_{\tilde{B}}}{\partial \tilde{\mu}_{d}}\right)_{p, \tilde{\mu}}-\xi_{\tilde{B}} & =0 .
\end{aligned}
$$

Using the Gibbs-Duhem thermodynamic relations (2.9) we can arrive at the expression

$$
d p=\frac{\epsilon+p}{T} d T+\rho T d \tilde{\mu}+\rho_{d} T d \tilde{\mu}_{d}
$$

which in turn can be easily cast into

$$
d T=\frac{T}{\epsilon+p} d p-\frac{\rho T^{2}}{\epsilon+p} d \tilde{\mu}-\frac{\rho_{d} T^{2}}{\epsilon+p} d \tilde{\mu}_{d}
$$

This provides the relations as follows:

$$
\left(\frac{\partial T}{\partial p}\right)_{\tilde{\mu}, \tilde{\mu}_{d}}=\frac{T}{\epsilon+p}, \quad\left(\frac{\partial T}{\partial \tilde{\mu}}\right)_{p, \tilde{\mu}_{d}}=-\frac{\rho T^{2}}{\epsilon+p}, \quad\left(\frac{\partial T}{\partial \tilde{\mu}_{d}}\right)_{p, \tilde{\mu}}=-\frac{\rho_{d} T^{2}}{\epsilon+p} .
$$

By virtue of (2.43) the first equations from each of the sets of the relations (2.32), (2.35) and (2.38), can be immediately integrated. The results yields

$$
D=T^{2} d\left(\tilde{\mu}, \tilde{\mu}_{d}\right), \quad D_{B}=T d_{B}\left(\tilde{\mu}, \tilde{\mu}_{d}\right), \quad D_{\tilde{B}}=T d_{\tilde{B}}\left(\tilde{\mu}, \tilde{\mu}_{d}\right),
$$

where $d_{i}=d\left(\tilde{\mu}, \tilde{\mu}_{d}\right), d_{B}\left(\tilde{\mu}, \tilde{\mu}_{d}\right), d_{\tilde{B}}\left(\tilde{\mu}, \tilde{\mu}_{d}\right)$ are the new functions, which do not depend on temperature $T$. Thus it is more convenient to treat $D_{i}$ as functions of temperature $T$, and chemical potentials $\tilde{\mu}$ and $\tilde{\mu}_{d}$.

To this end we assume the following dependence of the temperature $T=T\left(p, \tilde{\mu}, \tilde{\mu}_{d}\right)$ and use the relation

$$
\left(\frac{\partial D_{i}\left(T, \tilde{\mu}, \tilde{\mu}_{d}\right)}{\partial \tilde{\mu}}\right)_{p, \tilde{\mu}_{d}}=\left(\frac{\partial D_{i}\left(T, \tilde{\mu}, \tilde{\mu}_{d}\right)}{\partial \tilde{\mu}}\right)_{T, \tilde{\mu}_{d}}+\left(\frac{\partial D_{i}\left(T, \tilde{\mu}, \tilde{\mu}_{d}\right)}{\partial T}\right)_{\tilde{\mu}, \tilde{\mu}_{d}}\left(\frac{\partial T}{\partial \tilde{\mu}}\right)_{p, \tilde{\mu}_{d}} .
$$

The formula similar to (2.45) for the derivative with respect to $\tilde{\mu}_{d}$ is supposed. This leads to the system of differential equations provided by

$$
\begin{array}{r}
T\left(\frac{\partial D}{\partial T}\right)_{\tilde{\mu}, \tilde{\mu}_{d}}-2 D=0 \\
\left(\frac{\partial D}{\partial \tilde{\mu}}\right)_{T, \tilde{\mu}_{d}}-\frac{\rho T^{2}}{\epsilon+p}\left(\frac{\partial D}{\partial T}\right)_{\tilde{\mu}, \tilde{\mu}_{d}}-\xi=0, \\
\left(\frac{\partial D}{\partial \tilde{\mu}_{d}}\right)_{T, \tilde{\mu}}-\frac{\rho_{d} T^{2}}{\epsilon+p}\left(\frac{\partial D}{\partial T}\right)_{\tilde{\mu}, \tilde{\mu}_{d}}-\xi_{d}=0,
\end{array}
$$


and for $D_{B}$ one gets

$$
\begin{array}{r}
T\left(\frac{\partial D_{B}}{\partial T}\right)_{\tilde{\mu}, \tilde{\mu}_{d}}-D_{B}=0, \\
\left(\frac{\partial D_{B}}{\partial \tilde{\mu}}\right)_{T, \tilde{\mu}_{d}}-\frac{\rho T^{2}}{\epsilon+p}\left(\frac{\partial D_{B}}{\partial T}\right)_{\tilde{\mu} \cdot \tilde{\mu}_{d}}-\xi_{B}=0, \\
\left(\frac{\partial D_{B}}{\partial \tilde{\mu}_{d}}\right)_{T, \tilde{\mu}}-\frac{\rho_{d} T^{2}}{\epsilon+p}\left(\frac{\partial D}{\partial T}\right)_{\tilde{\mu}, \tilde{\mu}_{d}}-\xi_{\tilde{B} F}=0 .
\end{array}
$$

Consequently, one obtains the similar equations for $D_{\tilde{B}}$

$$
\begin{array}{r}
T\left(\frac{\partial D_{\tilde{B}}}{\partial T}\right)_{\tilde{\mu}, \tilde{\mu}_{d}}-D_{\tilde{B}}=0, \\
\left(\frac{\partial D_{\tilde{B}}}{\partial \tilde{\mu}}\right)_{T, \tilde{\mu}_{d}}-\frac{\rho T^{2}}{\epsilon+p}\left(\frac{\partial D_{\tilde{B}}}{\partial T}\right)_{\tilde{\mu}, \tilde{\mu}_{d}}-\xi_{F \tilde{B}}=0, \\
\left(\frac{\partial D_{\tilde{B}}}{\partial \tilde{\mu}_{d}}\right)_{T, \tilde{\mu}}-\frac{\rho_{d} T^{2}}{\epsilon+p}\left(\frac{\partial D_{\tilde{B}}}{\partial T}\right)_{\tilde{\mu}, \tilde{\mu}_{d}}-\xi_{\tilde{B}}=0 .
\end{array}
$$

To proceed, we shall replace the derivatives of the type $\left(\frac{\partial D_{i}}{\partial T}\right)_{\tilde{\mu}, \tilde{\mu}_{d}}$, combining relations resulting from the inspections of (2.46), (2.49), (2.52), and inserting them into the adequate equations (2.47)-(2.48), (2.50)-(2.51), (2.53)-(2.54), respectively. Consequently, we obtain the three sets of partial differential equations

$$
\begin{aligned}
\left(\frac{\partial D}{\partial \tilde{\mu}}\right)_{T, \tilde{\mu}_{d}} & =\frac{2 \rho T}{\epsilon+p} D+\xi=2 T D_{B} \\
\left(\frac{\partial D}{\partial \tilde{\mu}_{d}}\right)_{T, \tilde{\mu}} & =\frac{2 \rho_{d} T}{\epsilon+p} D+\xi_{d}=2 T D_{\tilde{B}}
\end{aligned}
$$

where the second equalities follow from the equations (2.23) and (2.24)

$$
\begin{aligned}
\left(\frac{\partial D_{B}}{\partial \tilde{\mu}}\right)_{T, \tilde{\mu}_{d}} & =\frac{\rho T}{\epsilon+p} D_{B}+\xi_{B}=C_{1} T \tilde{\mu} \\
\left(\frac{\partial D_{B}}{\partial \tilde{\mu}_{d}}\right)_{T, \tilde{\mu}} & =\frac{\rho_{d} T}{\epsilon+p} D_{B}+\xi_{\tilde{B} F}=C_{3} T \tilde{\mu}_{d}
\end{aligned}
$$

In the above derivations we use the relations (2.25) and (2.28). The last set of the equations can be easily achieved by incorporating (2.27) and (2.26). Namely, one has

$$
\begin{aligned}
\left(\frac{\partial D_{\tilde{B}}}{\partial \tilde{\mu}}\right)_{T, \tilde{\mu}_{d}} & =\frac{\rho T}{\epsilon+p} D_{\tilde{B}}+\xi_{F \tilde{B}}=C_{4} T \tilde{\mu}_{d}, \\
\left(\frac{\partial D_{\tilde{B}}}{\partial \tilde{\mu}_{d}}\right)_{T, \tilde{\mu}} & =\frac{\rho_{d} T}{\epsilon+p} D_{\tilde{B}}+\xi_{\tilde{B}}=C_{2} T \tilde{\mu} .
\end{aligned}
$$


The symmetry between the last two equations implies the equality $C_{2}=C_{4}$ and in the following we shall use the parameter $C_{2}$ only. It is customary to write the solutions of the aforementioned sets of the partial differential equations as follows [10]

$$
\begin{aligned}
D_{B} & =\frac{1}{2} C_{1} T \tilde{\mu}^{2}+\frac{1}{2} C_{3} T \tilde{\mu}_{d}^{2}+\gamma_{1}(T), \\
D_{\tilde{B}} & =C_{2} T \tilde{\mu} \tilde{\mu}_{d}+\gamma_{2}(T), \\
D & =\frac{1}{3} C_{1} T^{2} \tilde{\mu}^{3}+C_{2} T^{2} \tilde{\mu} \tilde{\mu}_{d}^{2}+2 \gamma_{1}(T) \tilde{\mu}+2 \gamma_{2}(T) \tilde{\mu}_{d}+\gamma_{3}(T),
\end{aligned}
$$

where $\gamma_{i}(T), i=1,2,3$ are temperature dependent functions. Using in the next step relations (2.46), (2.49) and (2.52), we achieve the following:

$$
\begin{aligned}
D_{B} & =\frac{1}{2} C_{1} T \tilde{\mu}^{2}+\frac{1}{2} C_{3} T \tilde{\mu}_{d}^{2}+\tilde{\gamma}_{1} T, \\
D_{\tilde{B}} & =C_{2} T \tilde{\mu} \tilde{\mu}_{d}+\tilde{\gamma}_{2} T, \\
D & =\frac{1}{3} C_{1} T^{2} \tilde{\mu}^{3}+C_{2} T^{2} \tilde{\mu} \tilde{\mu}_{d}^{2}+2 \tilde{\gamma}_{1} T^{2} \tilde{\mu}+2 \tilde{\gamma}_{2} T^{2} \tilde{\mu}_{d}+\tilde{\gamma}_{3} T^{2},
\end{aligned}
$$

where we have denoted by $\tilde{\gamma}_{i}, i=1,2,3$ integration constants.

Consequently, one can readily get the expressions for the novel kinetic coefficients of the forms

$$
\begin{aligned}
\xi= & C_{1} \mu^{2}\left(1-\frac{2}{3} \frac{\rho \mu}{\epsilon+p}\right)+\mu_{d}^{2} C_{2}\left(1-2 \frac{\rho \mu}{\epsilon+p}\right)-4 \frac{\rho T^{2}}{\epsilon+p}\left(\mu \tilde{\gamma}_{1}+\mu_{d} \tilde{\gamma}_{2}\right) \\
& -2 \frac{\rho T^{3}}{\epsilon+p} \tilde{\gamma}_{3}+2 \tilde{\gamma}_{1} T^{2} \\
\xi_{d}= & -\frac{2}{3} C_{1} \frac{\rho_{d} \mu^{3}}{\epsilon+p}+2 C_{2} \mu \mu_{d}\left(1-\frac{\rho_{d} \mu_{d}}{\epsilon+p}\right)-4 \frac{\rho_{d} T^{2}}{\epsilon+p}\left(\mu \tilde{\gamma}_{1}+\mu_{d} \tilde{\gamma}_{2}\right) \\
& -2 \frac{\rho_{d} T^{3}}{\epsilon+p} \tilde{\gamma}_{3}+2 \tilde{\gamma}_{2} T^{2} \\
\xi_{B}= & C_{1} \mu\left(1-\frac{1}{2} \frac{\rho \mu}{\epsilon+p}\right)-\frac{1}{2} C_{3} \frac{\rho \mu_{d}^{2}}{\epsilon+p}-\frac{\rho T^{2}}{\epsilon+p} \tilde{\gamma}_{1}, \\
\xi_{\tilde{B}}= & C_{2} \mu\left(1-\frac{\rho_{d} \mu_{d}}{\epsilon+p}\right)-\frac{\rho_{d} T^{2}}{\epsilon+p} \tilde{\gamma}_{2} . \\
\xi_{F \tilde{B}}= & C_{2} \mu_{d}\left(1-\frac{\rho \mu}{\epsilon+p}\right)-\frac{\rho T^{2}}{\epsilon+p} \tilde{\gamma}_{2}, \\
\xi_{\tilde{B} F}= & C_{3} \mu_{d}\left(1-\frac{1}{2} \frac{\rho_{d} \mu_{d}}{\epsilon+p}\right)-\frac{1}{2} C_{1} \frac{\rho_{d} \mu^{2}}{\epsilon+p}-\frac{\rho_{d} T^{2}}{\epsilon+p} \tilde{\gamma}_{1} .
\end{aligned}
$$

Equations (2.67)-(3.21) constitute the main results of the paper. They provide the generalization and in the appropriate limit reduce to those obtained earlier [5].

\section{Application to Dirac semi-metals with $\mathbb{Z}_{2}$ topological charge}

As was mentioned in the introduction most of the known Dirac semi-metals, in particular $\mathrm{Na}_{3} \mathrm{Bi}$ or $\mathrm{Cd}_{2} \mathrm{As}_{3}$, possess a chiral anomaly and two Dirac nodes, each carrying topological 
$\mathbb{Z}_{2}$ charge. In these materials two Dirac nodes are protected by rotational symmetry of the crystal. The two anomalies show up in our results as two different chemical potentials: $\mu$ corresponds to the chiral anomaly and its change results in the appearance of the chiral currents while $\mu_{d}$ decides about the position in energy of the two Dirac nodes. In the presence of a magnetic field parallel to an electric field the corresponding currents are not conserved. The current related to $\mathbb{Z}_{2}$ anomaly is a spin current, at least so, when the spin is approximately conserved [46].

First, let us analyze the obtained results for the kinetic coefficients in the system in question, in the light of the analog of chiral magnetic and chiral vortical effects. Roughly speaking by chiral magnetic effect one understands the induction of electromagnetic current by means of an external magnetic field to chiral media with non-vanishing chemical potentials [11]. On the other hand, the chiral vortical effect is bounded with an axial current in the direction of the local angular velocity [49].

Let us commence with the analog of the chiral magnetic effect in the system under consideration. One has two kinetic coefficients connected with $B^{(F) \mu}$, i.e., $\xi_{B}$ and $\xi_{\tilde{B} F}$. From the equation (2.69) it can be seen that although the kinetic coefficient is connected with the chiral magnetic effect responsible for ordinary Maxwell field it contains the influence of the auxiliary U(1)-gauge field. The relation (2.72) reveals also the dependence of both fields in the kinetic coefficient bounded with the chiral vortical effect. In the limit of square power of chemical potential and temperature tending to zero value, one arrives at the leading terms in magnetic chiral effect. Namely they yield

$$
\xi_{B}=C_{1} \mu+\mathcal{O}\left(\mu_{m}^{n \geq 2}, T\right), \quad \xi_{\tilde{B} F}=C_{3} \mu_{d}+\mathcal{O}\left(\mu_{m}^{n \geq 2}, T\right),
$$

and the leading term in chiral vortical effect is provided by

$$
\xi=C_{1} \mu^{2}+C_{2} \mu_{d}^{2}+\mathcal{O}\left(\mu_{m}^{n \geq 3}, T\right) .
$$

On the other hand, in the case of chiral vortical effect bounded with the auxiliary magnetic $\mathrm{U}(1)$-gauge field $\tilde{B}^{(B) \mu}$, there are two kinetic coefficients $\xi_{F \tilde{B}}$ and $\xi_{\tilde{B}}$ standing in front of the auxiliary magnetic field. Both equations (2.71) and (2.70), reveal that in the case under consideration we have the influence of the ordinary Maxwell field, as well as the additional one. The same situation is observed in $\xi_{d}$ coefficient, responsible for the chiral vortical effect. In the same limit as above, the leading terms describing chiral magnetic and chiral vortical effects connected with the auxiliary field are given, respectively by

$$
\xi_{F \tilde{B}}=C_{2} \mu_{d}+\mathcal{O}\left(\mu_{m}^{n \geq 2}, T\right), \quad \xi_{\tilde{B}}=C_{2} \mu+\mathcal{O}\left(\mu_{m}^{n \geq 2}, T\right),
$$

while the leading term in chiral vortical effect implies

$$
\xi_{d}=2 C_{2} \mu \mu_{d}+\mathcal{O}\left(\mu_{m}^{n \geq 3}, T\right) .
$$

Just in the system with chiral anomaly and $\mathbb{Z}_{2}$ anomalous topological charge we obtained four kinetic coefficients that exhibit chiral magnetic effect $\left(\xi_{B}, \xi_{\tilde{B} F}, \xi_{F \tilde{B}}, \xi_{\tilde{B}}\right)$, and two which determine chiral vortical effect $\left(\xi, \xi_{d}\right)$. 
Inspection of the equations defining currents $j_{\alpha}(F)$ and $j_{\alpha}(B)$, as well as, the definitions of $V_{F}^{\alpha}$ and $V_{B}^{\alpha}(2.15)-(2.16)$, reveal that the Maxwell field current is connected with the following kinetic coefficients $\left(\xi, \xi_{B}, \xi_{F \tilde{B}}\right)$, while $j_{\alpha}(B)$ is bounded with $\left(\xi_{d}, \xi_{\tilde{B}}, \xi_{\tilde{B} F}\right)$. In turn it leads to the conclusion that both $j_{\alpha}(F)$ and $j_{\alpha}(B)$ have terms depending on chiral magnetic effect, i.e., $j_{\alpha}(F) \sim\left(\xi_{B}, \xi_{F \tilde{B}}\right), j_{\alpha}(B) \sim\left(\xi_{\tilde{B}}, \xi_{\tilde{B} F}\right)$, and chiral vortical one, $j_{\alpha}(F) \sim$ $\xi, j_{\alpha}(B) \sim \xi_{d}$. The result in question generalizes the relations on the currents obtained in [46], equations (13)-(14), because of the fact that the kinetic coefficients determined by the (2.67)-(2.72) contain both the influence of the ordinary Maxwell field characteristics like $\rho, \mu$, and the auxiliary $\mathrm{U}(1)$-gauge field attributes, $\rho_{d}, \mu_{d}$. The other bonus is the temperature dependence of them.

Let us give some remarks concerning the strength of both $\mathrm{U}(1)$-gauge fields and their influence on the obtained kinetic coefficients. In the case when $\rho$ and $\mu$ of the ordinary Maxwell field are far more greater than those for the auxiliary gauge field responsible for $\mathbb{Z}_{2}$ anomaly, we obtain

$$
\begin{aligned}
\xi & =C_{1} \mu^{2}\left(1-\frac{2}{3} \frac{\rho \mu}{\epsilon+p}\right)-4 \frac{\rho T^{2}}{\epsilon+p} \mu \tilde{\gamma}_{1}-2 \frac{\rho T^{3}}{\epsilon+p} \tilde{\gamma}_{3}+2 \tilde{\gamma}_{1} T^{2} \\
\xi_{d} & =2 \tilde{\gamma}_{2} T^{2} \\
\xi_{B} & =C_{1} \mu\left(1-\frac{1}{2} \frac{\rho \mu}{\epsilon+p}\right)-\frac{\rho T^{2}}{\epsilon+p} \tilde{\gamma}_{1}, \\
\xi_{\tilde{B}} & =C_{2} \mu \\
\xi_{F \tilde{B}} & =-\frac{\rho T^{2}}{\epsilon+p} \tilde{\gamma}_{2} \\
\xi_{\tilde{B} F} & =0
\end{aligned}
$$

On the other hand, in the opposite case when $\rho$ and $\mu$ tend to zero, one receives

$$
\begin{aligned}
\xi & =\mu_{d}^{2} C_{2}-4 \frac{\rho T^{2}}{\epsilon+p} \mu_{d} \tilde{\gamma}_{2}+2 \tilde{\gamma}_{1} T^{2}, \\
\xi_{d} & =-4 \frac{\rho_{d} T^{2}}{\epsilon+p} \mu_{d} \tilde{\gamma}_{2}-2 \frac{\rho_{d} T^{3}}{\epsilon+p} \tilde{\gamma}_{3}+2 \tilde{\gamma}_{2} T^{2}, \\
\xi_{B} & =0 \\
\xi_{\tilde{B}} & =-\frac{\rho_{d} T^{2}}{\epsilon+p} \tilde{\gamma}_{2} . \\
\xi_{F \tilde{B}} & =C_{2} \mu_{d}, \\
\xi_{\tilde{B} F} & =C_{3} \mu_{d}\left(1-\frac{1}{2} \frac{\rho_{d} \mu_{d}}{\epsilon+p}\right)-\frac{1}{2} C_{1} \frac{\rho_{d} \mu^{2}}{\epsilon+p}-\frac{\rho_{d} T^{2}}{\epsilon+p} \tilde{\gamma}_{1} .
\end{aligned}
$$

In the first limiting case, one can observe that up to the leading terms $\mathcal{O}\left(\mu_{m}^{n \geq 3}, T\right)$, the chiral vortical effect is connected with $\xi \sim C_{1} \mu^{2}$, while for the chiral magnetic effect, $\xi_{B} \sim C_{1} \mu$ and $\xi_{\tilde{B}} \sim C_{2} \mu$ kinetic coefficients, are responsible. On the other hand, when $\rho$, $\mu \rightarrow 0$, the chiral vortical effect is bounded with $\xi \sim C_{2} \mu_{d}^{2}$, and the chiral magnetic effect is connected with $\xi_{F \tilde{B}} \sim C_{2} \mu_{d}$ and $\xi_{\tilde{B} F} \sim C_{3} \mu_{d}$. 
Of course, one can see that both anomalies interplay will exert its influence on the magneto-transport properties, as was pointed out in [46]. The forms of the currents obtained in the aforementioned paper, i.e., their dependence on chiral magnetic and vortical effects played the crucial role in the experimentally verifiable effects of magneto-transport.

In our case we obtained far more richer structure of the kinetic coefficients bounded with the adequate currents, so we conclude that the magnetic conductivity will be modified by parameters of both considered fields and temperature.

Due to this interpretation of the $\mathbb{Z}_{2}$ bound current, one expects that spin - related magnetic field $\tilde{B}_{\alpha}^{(B)}$ vanishes [46]. This requirement causes that in the equation (2.15) there is no term with $\xi_{F \tilde{B}}$, and the relation (2.16) is lack of $\xi_{\tilde{B}}$. Consequently we get the following kinetic coefficients:

$$
\begin{aligned}
\xi= & C_{1} \mu^{2}\left(1-\frac{2}{3} \frac{\rho \mu}{\epsilon+p}\right)+\mu_{d}^{2} C_{2}\left(1-2 \frac{\rho \mu}{\epsilon+p}\right)-4 \frac{\rho T^{2}}{\epsilon+p}\left(\mu \tilde{\gamma}_{1}+\mu_{d} \tilde{\gamma}_{2}\right) \\
& -2 \frac{\rho T^{3}}{\epsilon+p} \tilde{\gamma}_{3}+2 \tilde{\gamma}_{1} T^{2}, \\
\xi_{d}= & -\frac{2}{3} C_{1} \frac{\rho_{d} \mu^{3}}{\epsilon+p}+2 C_{2} \mu \mu_{d}\left(1-\frac{\rho_{d} \mu_{d}}{\epsilon+p}\right)-4 \frac{\rho_{d} T^{2}}{\epsilon+p}\left(\mu \tilde{\gamma}_{1}+\mu_{d} \tilde{\gamma}_{2}\right) \\
& -2 \frac{\rho_{d} T^{3}}{\epsilon+p} \tilde{\gamma}_{3}+2 \tilde{\gamma}_{2} T^{2}, \\
\xi_{B}= & C_{1} \mu\left(1-\frac{1}{2} \frac{\rho \mu}{\epsilon+p}\right)-\frac{1}{2} C_{3} \frac{\rho \mu_{d}^{2}}{\epsilon+p}-\frac{\rho T^{2}}{\epsilon+p} \tilde{\gamma}_{1}, \\
\xi_{\tilde{B} F}= & C_{3} \mu_{d}\left(1-\frac{1}{2} \frac{\rho_{d} \mu_{d}}{\epsilon+p}\right)-\frac{1}{2} C_{1} \frac{\rho_{d} \mu^{2}}{\epsilon+p}-\frac{\rho_{d} T^{2}}{\epsilon+p} \tilde{\gamma}_{1} .
\end{aligned}
$$

It is worth pointing out that even in the absence of $\tilde{B}^{(B) \alpha}$ field (but presence of $\tilde{E}^{(B) \alpha}$ ), one obtains two non-vanishing kinetic coefficients $\xi$ and $\xi_{d}$, which exhibit the chiral vortical effect. They are functions of both chemical potentials and density currents, as well as, temperature. In comparison to the case studied in [5], one obtains $\xi_{d}$ kinetic coefficient, connected with spin conductivity (and possibly the spin Hall effect) which is affected by both U(1)-gauge fields. Moreover the absence of $\tilde{B}^{(B) \alpha}$ induces the chiral magnetic effects, described by $\xi_{B}$ and $\xi_{\tilde{B} F}$ coefficients. In $\xi_{B}$, the $C_{3}$ parameter modifies the kinetic coefficient, which constitutes the novelty comparing to the previously studied case $[5,10]$. It contains the influence of the auxiliary gauge field, its electric component. The novelty is bounded also with $\xi_{\tilde{B} F}$, which mostly depends on $\rho_{d}, \mu_{d}$, but also includes the influence of $\mu^{2}$.

These findings harmonize with the recent kinetic calculations [46], where the authors have noted that the $\mathbb{Z}_{2}$ anomaly affects magneto-transport properties of Dirac semi-metals. The observational manifestation of the $\mathbb{Z}_{2}$ anomaly found earlier is connected with the reduction of the diagonal resistivity due to the spin Hall effect and the narrowing of the angular dependence of the magneto-resistance. The detailed analysis of the magnetoconductivity and magneto-resistivity of the Weyl semi-metals based on the presented hydrodynamic approach [50] will be presented in the future publication. 


\section{Summary and conclusions}

We have examined the generalized equations of relativistic hydrodynamics allowing the description of electrons in condensed matter systems with linear spectrum and the two different types of anomalies. One of them is the well known chiral anomaly, while the other one, authorizes the anomaly observed in one class of Dirac semi-metal characterized by two Dirac nodes separated in momentum space and lying on the axis of rotation. With the $\mathbb{Z}_{2}$ anomaly the corresponding charge density $\rho_{d}$ is connected. Its existence forces the non-trivial generalization of the relativistic hydrodynamics.

We have found that the additional kinetic parameters, bounded with two different anomalous charges and required by the second law of thermodynamics and positiveness of the entropy production during the flow of electron fluid, enter the hydrodynamic equations in the similar manner. They are a source of the additional kinetic coefficients called earlier magnetic conductivities. In fact these are spin and spin Hall conductivities [46]. Their appearance in the hydrodynamic equations can be traced back to the necessity of adding dissipative terms proportional to the vorticity and magnetic components of the two U(1)gauge fields. Up to the first order in the velocity gradients, they constitute the important component in the proper description of the relativistic fluid.

In the case under considerations we obtain two kinetic coefficients exhibiting the chiral vortical effect, $\xi$ and $\xi_{d}$, and two describing chiral magnetic effects. Interestingly the existence of $\mathbb{Z}_{2}$ anomaly induces kinetic coefficient $\xi_{d}$ connected with the $\mathbb{Z}_{2}$ related conductivity. We argue that this conductivity is connected with spin conductivity and spin Hall effect in the kinetic approach to the problem in question. The $\xi$ and $\xi_{d}$ coefficients are dependent on chemical potentials of both fields, their densities and temperature.

In the model under consideration, the absence of auxiliary magnetic field induces two kinetic coefficients bounded with the chiral magnetic effect $\left(\xi, \xi_{\tilde{B} F}\right)$. In $\xi_{d}$ the existence of $C_{3}$ parameter modifies the previously obtained results. On the other hand, $\xi_{\tilde{B} F}$ depends mostly on the quantities characterizing the additional gauge field. This finding provides the generalization of the previous work on hydrodynamics with quantum triangle anomalies [5].

Let us discuss some possible experimental evidences of the theory in question. From the hydrodynamical calculations one obtains new formulae for the kinetic coefficients which constitute the vital ingredient of the currents connected with Maxwell and the auxiliary $\mathrm{U}(1)$-gauge fields. They reveal the dependence on temperature, chiral magnetic and vortical effects. On the other hand they are functions of characteristics of both fields. In order to fully explore the validity of the hydrodynamic theory in real materials being type II Dirac semimetals, one has to calculate the transport matrix $\sigma_{(F, B, F \tilde{B}, B F)}$ for a system in weak electric and parallel to it magnetic fields. The hydrodynamic theory provides a general information about the positivity of the matrix, but to the given accuracy does not fix it.

The possible experimental verifications of the presented theory might consist of the measurements (similar to those provided in [39]) of the dependence of magnetic conductivities on the parameters bounded with the gauge fields and constants $C_{i}$, related to the adequate type of anomaly. Such experiments might put some experimental restrictions on the aforementioned parameters. Basing on the kinetic theory presented in [46], one expects 
that additional anomaly should lead to the narrowing of the magneto-conductivity curve. This will be studied in the forthcoming paper, in which we shall calculate the conductivity matrix $\sigma_{(F, B, F \tilde{B}, B F)}$ by hydrodynamic theory and also using holography. The preliminary results show that in our approach the presence of $\mathbb{Z}_{2}$ anomaly likewise leads to narrowing of the magneto-conductivity line. The narrowing of the magneto-conductivity curve and its dependence on temperature were confirmed by the recent experimental data $[24,28,29,37]$.

\section{Acknowledgments}

MR and KIW were partially supported by the grant DEC-2014/15/B/ST2/00089 of the National Science Center. We would like to thank the Referee for valuable comments and remarks.

Open Access. This article is distributed under the terms of the Creative Commons Attribution License (CC-BY 4.0), which permits any use, distribution and reproduction in any medium, provided the original author(s) and source are credited.

\section{References}

[1] L.D. Landau and E.M. Lifshitz, Fluid Mechanics, Pergamon, New York U.S.A. (1959).

[2] C. Eckart, The Thermodynamics of irreversible processes. III. Relativistic theory of the simple fluid, Phys. Rev. 58 (1940) 919 [InSPIRE].

[3] S.L. Adler, Axial vector vertex in spinor electrodynamics, Phys. Rev. 177 (1969) 2426 [INSPIRE].

[4] J.S. Bell and R. Jackiw, A PCAC puzzle: $\pi^{0} \rightarrow \gamma \gamma$ in the $\sigma$ model, Nuovo Cim. A 60 (1969) 47 [inSPIRE].

[5] D.T. Son and P. Surówka, Hydrodynamics with Triangle Anomalies, Phys. Rev. Lett. 103 (2009) 191601 [arXiv:0906.5044] [INSPIRE].

[6] M. Lublinsky and I. Zahed, Anomalous Chiral Superfluidity, Phys. Lett. B 684 (2010) 119 [arXiv: 0910.1373] [INSPIRE].

[7] S. Lin, On the anomalous superfluid hydrodynamics, Nucl. Phys. A 873 (2012) 28 [arXiv: 1104.5245] [INSPIRE].

[8] J. Bhattacharya, S. Bhattacharyya, S. Minwalla and A. Yarom, A Theory of first order dissipative superfluid dynamics, JHEP 05 (2014) 147 [arXiv:1105.3733] [INSPIRE].

[9] C. Eling, Y. Neiman and Y. Oz, Holographic Non-Abelian Charged Hydrodynamics from the Dynamics of Null Horizons, JHEP 12 (2010) 086 [arXiv:1010.1290] [INSPIRE].

[10] Y. Neiman and Y. Oz, Relativistic Hydrodynamics with General Anomalous Charges, JHEP 03 (2011) 023 [arXiv: 1011.5107] [INSPIRE].

[11] D. Kharzeev, Parity violation in hot QCD: Why it can happen and how to look for it, Phys. Lett. B 633 (2006) 260 [hep-ph/0406125] [INSPIRE].

[12] D.E. Kharzeev, The Chiral Magnetic Effect and Anomaly-Induced Transport, Prog. Part. Nucl. Phys. 75 (2014) 133 [arXiv:1312.3348] [INSPIRE]. 
[13] K. Fukushima, D.E. Kharzeev and H.J. Warringa, The Chiral Magnetic Effect, Phys. Rev. D 78 (2008) 074033 [arXiv: 0808.3382] [InSPIRE].

[14] D.E. Kharzeev and H.-U. Yee, Anomalies and time reversal invariance in relativistic hydrodynamics: the second order and higher dimensional formulations, Phys. Rev. D 84 (2011) 045025 [arXiv: 1105.6360] [INSPIRE].

[15] I. Gahramanov, T. Kalaydzhyan and I. Kirsch, Anisotropic hydrodynamics, holography and the chiral magnetic effect, Phys. Rev. D 85 (2012) 126013 [arXiv:1203.4259] [INSPIRE].

[16] M.V. Isachenkov and A.V. Sadofyev, The Chiral magnetic effect in hydrodynamical approach, Phys. Lett. B 697 (2011) 404 [arXiv: 1010.1550] [INSPIRE].

[17] H.B. Nielsen and M. Ninomiya, Adler-Bell-Jackiw anomaly and Weyl fermions in crystal, Phys. Lett. B 130 (1983) 389 [InSPIRE].

[18] D.T. Son and B.Z. Spivak, Chiral Anomaly and Classical Negative Magnetoresistance of Weyl Metals, Phys. Rev. B 88 (2013) 104412 [arXiv:1206.1627] [INSPIRE].

[19] A.A. Burkov, Negative longitudinal magnetoresistance in Dirac and Weyl metals, Phys. Rev. B 91 (2015) 245157.

[20] Y. Bu, R.-G. Cai, Q. Yang and Y.-L. Zhang, Holographic Charged Fluid with Chiral Electric Separation Effect, arXiv:1803.08389 [INSPIRE].

[21] A.H. Castro Neto, F. Guinea, N.M.R. Peres, K.S. Novoselov and A.K. Geim, The electronic properties of graphene, Rev. Mod. Phys. 81 (2009) 109 [inSPIRE].

[22] M.Z. Hasan and C.L. Kane, Topological Insulators, Rev. Mod. Phys. 82 (2010) 3045 [arXiv: 1002.3895] [INSPIRE].

[23] Z.K. Liu et al., Discovery of a three-dimensional topological Dirac semimetal, $\mathrm{Na} \mathrm{a}_{3} \mathrm{Bi}$, Science 343 (2014) 864.

[24] S.Y. Xu et al., Observation of Fermi arc surface states in a topological metal, Science 347 (2015) 294.

[25] Z.K. Liu et al., A stable three-dimensional topological Dirac semimetal $C d_{3} A s_{2}$, Nat. Mater. 13 (2014) 677.

[26] H.-Z. Lu and S.-Q. Shen, Quantum transport in topological semimetals under magnetic fields, Front. Phys. 12 (2017) 127201.

[27] R. Lundgren, P. Laurell and G.A. Fiete, Thermoelectric properties of Weyl and Dirac semimetals, Phys. Rev. B 90 (2014) 165115 [arXiv:1407.1435] [INSPIRE].

[28] M. Neupane et al., Observation of a three-dimensional topological Dirac semimetal phase in high-mobility $\mathrm{Cd}_{3} \mathrm{As}_{2}$, Nat. Commun. 5 (2014) 3786.

[29] M. Neupane et al., Observation of topological nodal fermion semimetal phase in ZrSiS, Phys. Rev. B 93 (2016) 201104(R) [arXiv:1604.00720].

[30] M.S. Foster and I.L. Aleiner, Slow imbalance relaxation and thermoelectric transport in graphene, Phys. Rev. B 79 (2009) 085415.

[31] J. Crossno et al., Observation of the Dirac fluid and the breakdown of the Wiedemann-Franz law in graphene, Science 351 (2016) 1058 [arXiv: 1509.04713].

[32] T. Liang, Q. Gibson, M.N. Ali, M. Liu, R.J. Cava and N.P. Ong, Ultrahigh mobility and giant magnetoresistance in the Dirac semimetal $\mathrm{Cd}_{3} A s_{2}$, Nat. Mater. 14 (2015) 280. 
[33] T. Liang et al., Anomalous Nernst Effect in the Dirac Semimetal $\mathrm{Cd}_{3} A s_{2}$, Phys. Rev. Lett. 118 (2017) 136601 [arXiv:1610.02459] [INSPIRE].

[34] L.-P. He and S.-Y. Li, Quantum transport properties of the three-dimensional Dirac semimetal $C d_{3} A s_{2}$ single crystals, Chin. Phys. B 25 (2016) 117105.

[35] J. Xiong et al., Evidence for the chiral anomaly in the Dirac semimetal $\mathrm{Na}_{3} \mathrm{Bi}$, Science 350 (2015) 413.

[36] Q. Li et al., Chiral magnetic effect in $Z r T e_{5}$, Nat. Phys. 12 (2016) 550.

[37] C. Zhang et al., Observation of the Adler-Bell-Jackiw chiral anomaly in a Weyl semimetal, arXiv: 1503.02630 [INSPIRE].

[38] X. Huang et al., Observation of the Chiral-Anomaly-Induced Negative Magnetoresistance in 3D Weyl Semimetal TaAs, Phys. Rev. X 5 (2015) 031023.

[39] J. Gooth et al., Experimental signatures of the mixed axial-gravitational anomaly in the Weyl semimetal NbP, Nature $\mathbf{5 4 7}$ (2017) 324 [arXiv: 1703.10682] [INSPIRE].

[40] S.M. Young, S. Zaheer, J.C.Y. Teo, C.L. Kane, E.J. Mele and A.M. Rappe, Dirac semimetal in three dimensions, Phys. Rev. Lett. 108 (2012) 140405.

[41] C.K. Chiu, J.C.Y. Teo, A.P. Schnyder and S. Ryu, Classification of topological quantum matter with symmetries, Rev. Mod. Phys. 88 (2016) 035005.

[42] B.J. Yang and N. Nagaosa, Classification of stable three-dimensional Dirac semimetals with nontrivial topology, Nat. Commun. 5 (2014) 4898.

[43] B.J. Yang, T. Morimoto and A. Furusaki, Topological charges of three-dimensional Dirac semimetals with rotation symmetry, Phys. Rev. B 92 (2015) 165120.

[44] C. Fang, Y. Chen, H.Y. Kee and L. Fu, Topological nodal line semimetals with and without spin-orbital coupling, Phys. Rev. B 92 (2015) 081201 [arXiv:1506.03449].

[45] S. Kobayashi and M. Sato, Topological Superconductivity in Dirac Semimetals, Phys. Rev. Lett. 115 (2015) 187001 [arXiv:1504.07408] [INSPIRE].

[46] A.A. Burkov and Y.B. Kim, $\mathcal{Z}_{2}$ and Chiral Anomalies in Topological Dirac Semimetals, Phys. Rev. Lett. 117 (2016) 136602.

[47] Y. Neiman and Y. Oz, Relativistic Hydrodynamics with General Anomalous Charges, JHEP 03 (2011) 023 [arXiv: 1011.5107] [INSPIRE].

[48] A. Lucas, R.A. Davison and S. Sachdev, Hydrodynamic theory of thermoelectric transport and negative magnetoresistance in Weyl semimetals, Proc. Natl. Acad. Sci. Unit States Am. 113 (2016) 9463.

[49] D.E. Kharzeev and D.T. Son, Testing the chiral magnetic and chiral vortical effects in heavy ion collisions, Phys. Rev. Lett. 106 (2011) 062301 [arXiv: 1010.0038] [INSPIRE].

[50] M. Rogatko and K.I. Wysokinski, work in progress. 